\title{
Development of Nanostructured Antireflection Coatings for EO/IR Sensor and Solar Cell Applications
}

\author{
Ashok K. Sood ${ }^{1,2}$, Adam W. Sood ${ }^{1,2,3}$, Roger E. Welser ${ }^{1,2}$, Gopal G. Pethuraja ${ }^{1,2,4}$, Yash R. Puri ${ }^{1,2}$, \\ Xing Yan , David J. Poxson $^{3}$, Jaehee Cho ${ }^{3}$, E. Fred Schubert ${ }^{3}$, Nibir K. Dhar ${ }^{5}$, Dennis L. Polla ${ }^{5,6}$, \\ Pradeep Haldar ${ }^{4}$, Jennifer L. Harvey ${ }^{7}$
}

${ }^{1}$ Magnolia Solar Inc., Albany, NY, USA; ${ }^{2}$ Magnolia Optical Technologies Inc., Woburn, MA, USA; ${ }^{3}$ Department of Electrical and Computer Systems Engineering and Physics, Rensselaer Polytechnic Institute, Troy, NY, USA; ${ }^{4}$ College of Nanoscale Science and Engineering, Albany, NY, USA; ${ }^{5}$ Microsystems Technology Office of the Defense Advanced Research Projects Agency, Arlington, VA, USA; ${ }^{6}$ College of Science and Engineering, University of Minnesota, Minneapolis, MN, USA; ${ }^{7}$ New York State Energy Research and Development Authority, Albany, NY, USA.

Email: aksood@magnoliasolar.com

Received May $17^{\text {th }}, 2012$; revised June $18^{\text {th }}, 2012$; accepted July $21^{\text {st }}, 2012$

\begin{abstract}
Electro-optical/infrared (EO/IR) sensors and photovoltaic power sources are being developed for a variety of defense and commercial applications. One of the critical technologies that will enhance both EO/IR sensor and photovoltaic module performance is the development of high quality nanostructure-based antireflection coatings. In this paper, we review our work on advanced antireflection structures that have been designed by using a genetic algorithm and fabricated by using oblique angle deposition. The antireflection coatings are designed for the wavelength range of $250 \mathrm{~nm}$ to $2500 \mathrm{~nm}$ and an incidence angle between $0^{\circ}$ and $40^{\circ}$. These nanostructured antireflection coatings are shown to enhance the optical transmission through transparent windows over a wide band of interest and minimize broadband reflection losses to less than one percent, a substantial improvement over conventional thin-film antireflection coating technologies.
\end{abstract}

Keywords: Antireflection Coating; Nanostructured Layer; Oblique Angle Deposition; Silicon Dioxide; Broadband; Omni-Directional

\section{Introduction}

EO/IR sensors are being developed for a variety of defense and commercial systems applications [1-8]. These include ultraviolet (UV), visible, near infrared (NIR), mid-wavelength infrared (MWIR), and long-wavelength infrared (LWIR) nanotechnology-based sensors. Visible and NIR focal plane arrays (FPAs) typically use InGaAs or SiGe absorber materials that operate in the 0.4 to 1.7 micron band [2,3]. MWIR sensors, on the other hand, use InSb- or HgCdTe-based FPAs that are sensitive in the 3 - 5 micron region [4,5]. To access the LWIR band, EO/IR sensors employ either $\mathrm{HgCdTe}$ materials, strained-layer super lattice (SLS) structures, or Si-MEMS based microbolometers $[6,7]$. To further enhance the performance of EO/IR sensors, radiation hardened antireflection coatings have been studied, using multilayer coatings on silicon and CdTe substrates for MWIR and LWIR applications [8].

One of the critical technologies that will further improve $\mathrm{EO} / \mathrm{IR}$ sensor performance is the development of high quality nanostructure-based antireflection coatings (ARCs). Similar high performance ARCs are also needed to im- prove the power output of photovoltaic modules. In this paper, we review our efforts to develop nanostructured ARCs by employing an oblique angle nanowire growth technique. The antireflection coatings are designed for the wavelength range of $250 \mathrm{~nm}$ to $2500 \mathrm{~nm}$ and an incidence angle between $0^{\circ}$ and $40^{\circ}$. Nanostructure-based ARCs can enhance the performance of UV, visible, and NIR sensors, and extend their utility to longer wavelength applications.

\section{Oblique Angle Deposition}

The use of porous nanostructured materials to minimize reflection losses has recently been studied by Prof. Fred Schubert's group at the Rensselaer Polytechnic Institute (RPI) [9]. Their work has explored the possibility of using nanowires grown by the oblique angle deposition technique to create advanced antireflection structures. Their investigation was motivated by the classic work of Lord Rayleigh on graded-refractive index layers to achieve broadband and omnidirectional antireflection characteristics.

Graded-index coatings with different index profiles have been theoretically investigated for their broadband antire- 
flection properties, particularly with air as the ambient medium. However, because of the unavailability of optical materials with very low refractive indices that closely match the refractive index of air, such high quality broadband antireflection coatings have not been realizable. The work carried out by Xi et al. [10] used titanium dioxide $\left(\mathrm{TiO}_{2}\right)$ and silicon dioxide $\left(\mathrm{SiO}_{2}\right)$ graded-index nanowires and nanorods deposited by oblique-angle deposition, and, for the first time, demonstrated their potential for antireflection coatings by virtually eliminating Fresnel reflection from an aluminum nitride (AIN)/air interface over the UV band. This was achieved by controlling the refractive index of the $\mathrm{TiO}_{2}$ and $\mathrm{SiO}_{2}$ nanorod layers, down to a minimum value of $n=1.05$, the lowest value so far reported [10].

Figure 1 presents the detailed layout of the experimental set-up of the oblique angle deposition technique for growth of porous nanostructured materials by e-beam evaporation. This technique creates a highly directional vapor flux and can be implemented with a variety of optical coating materials. Oblique-angle deposition is a method of growing porous thin-films, and hence thin-films with lowrefractive index, enabled by surface diffusion and selfshadowing effects during the deposition process. Random growth fluctuations on the substrate produce a shadow region that incident vapor flux cannot reach, and a nonshadow region where incident flux deposits preferentially, thereby creating an oriented rod-like structure with high porosity, as illustrated in Figure 2. The deposition angle, defined as the angle between the normal to the sample surface and the incident vapor flux, results in the formation of nanorod structures that are tilted relative to the sample surface.

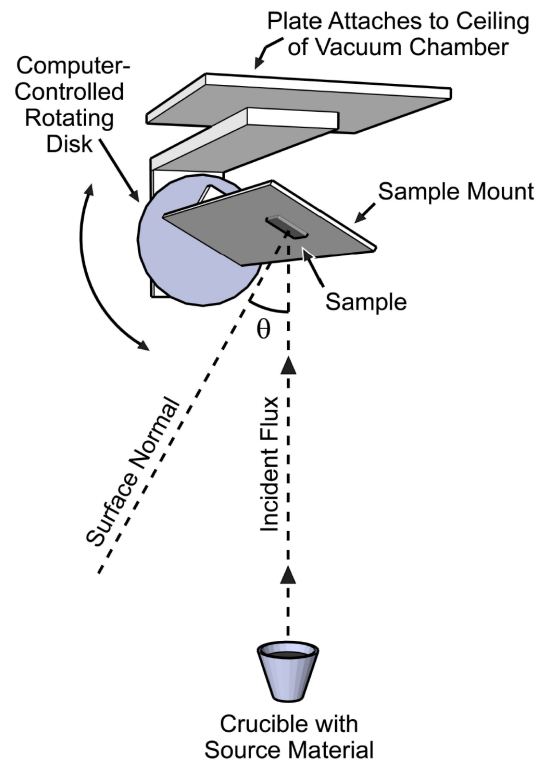

Figure 1. Illustration of the experimental setup used in the oblique-angle deposition technique for porous nanostructured materials. The incident physical vapor flux strikes the sample at an angle $\theta$ relative to the surface normal.
Oblique angle deposition is a self-organized process that can be applied to many different materials. Figure 3, for example, depicts cross-sectional scanning electron micrographs of two nanostructured thin films: one employing $\mathrm{SiO}_{2}$ material and the other indium tin oxide (ITO). Both films were deposited at highly oblique angles $\left(\sim 80^{\circ}\right)$, which resulted in the formation of well defined nanorod structures.

Because the gaps between the nanorods can be much smaller than the wavelength of visible and infrared light, the nanostructured layers typically act as a single homogenous film with a refractive index intermediate between air and the nanorod material, decreasing in refractive index with increasing porosity. Figure 4 presents the measured refractive index dispersion curve as a function of wavelength from a layer of nanostructured $\mathrm{SiO}_{2}$ deposited at a highly oblique angle [10]. This low index nanostructured $\mathrm{SiO}_{2}$ film was deposited on a silicon substrate and measured by ellipsometry. Also shown is a comparison of experimental reflectivity data with theoretical calculations. These results illustrate that nanowires and nanorods grown by the oblique angle deposition technique provide a pathway for fabricating high-quality broadband anti-reflection coatings for a variety of nanosensor applications.

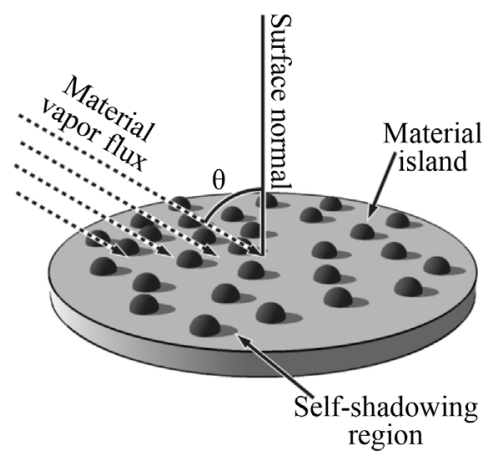

(a)

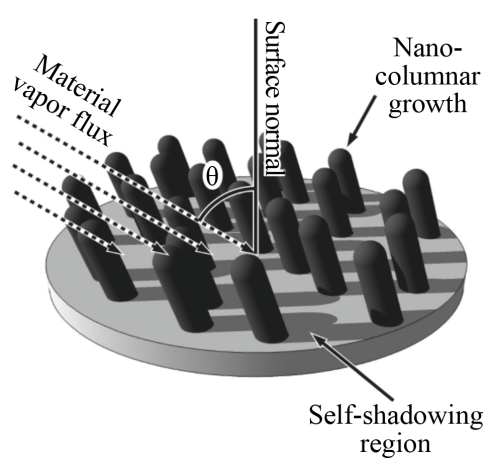

(b)

Figure 2. Simplified schematic of the oblique-angle deposition process for synthesizing porous, nanostructured films, showing (a) The initial formation of material islands at random locations across the substrate, followed by (b) The formation of self-shadowed regions and nano-columnar growth when material vapor flux arrives at a non-normal deposition angle $(\theta)$ to the substrate. 


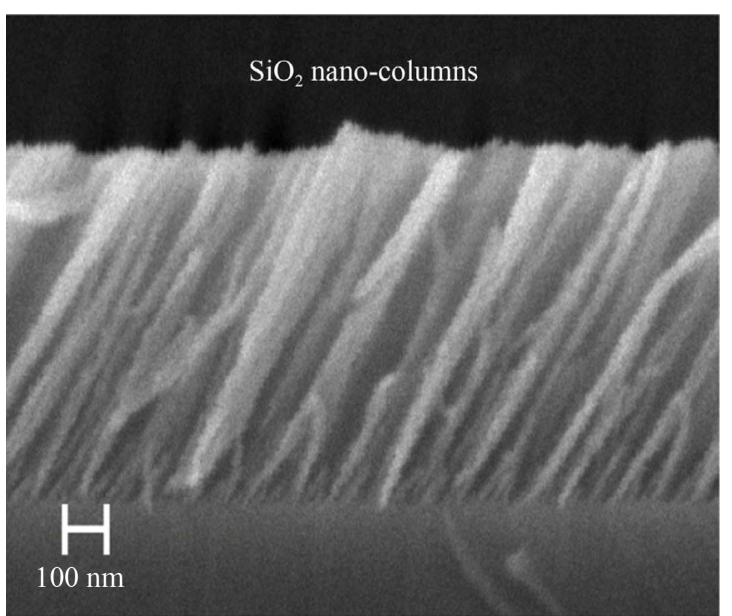

(a)

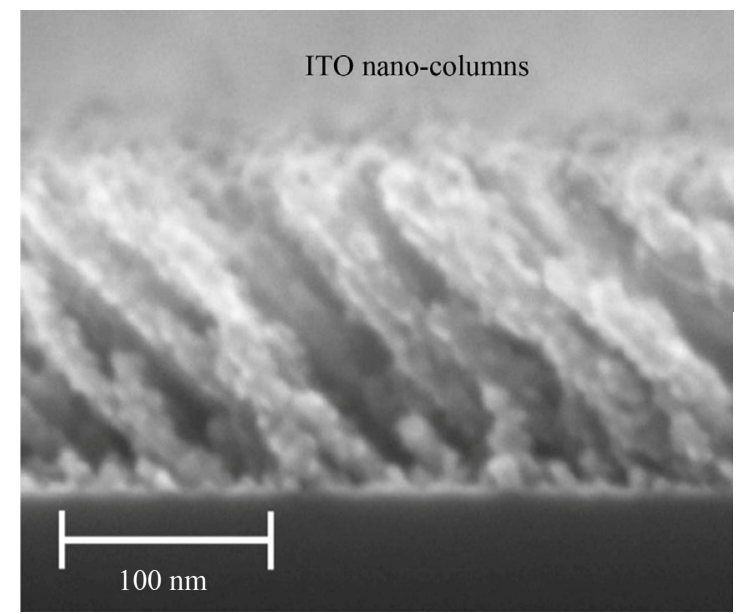

(b)

Figure 3. Cross-sectional scanning electron micrographs of nanostructured optical thin-films deposited by oblique angle deposition using (a) Silicon dioxide and (b) Indium tin oxide materials.

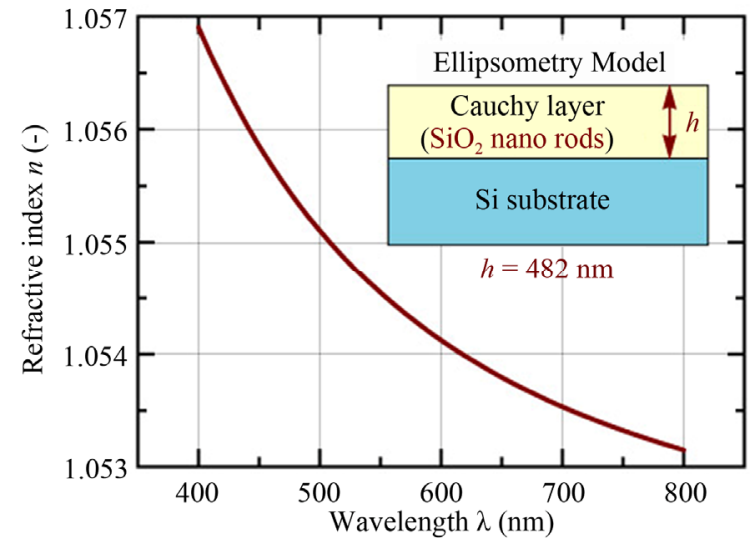

(a)

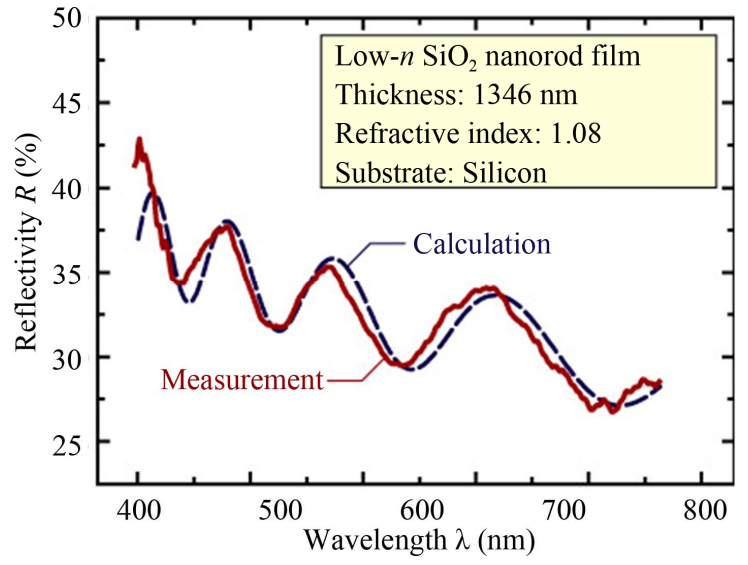

(b)

Figure 4. (a) Refractive index dispersion curve of a low index $\mathrm{SiO}_{2}$ nanorod thin film on a silicon substrate as measured by ellipsometry with (b) A comparison of the measured and calculated reflectivity spectrum.

Figures 5 and 6 demonstrate the use of $\mathrm{SiO}_{2}$ and $\mathrm{TiO}_{2}$ nanowire and nanorods to achieve a high performance, step-graded antireflection coating on an AlN substrate. The feasibility of this technology has been demonstrated for UV light-emitting diode (LED) applications [10]. In this paper, we summarize our recent efforts to extend this technology to other substrates and other bands of interest in the visible, NIR, and MWIR spectrums for next generation EO/IR sensors.

\section{Modeling of Nanostructured Antireflection Coatings}

We have applied a genetic algorithm to optimize antireflection coatings for optical sensors with air as the ambient medium. These calculations can consider multi-layer coatings composed of co-sputtered and porous nanostructured materials and take material dispersion into account
[11]. In particular, each layer may be composed of either nano-porous $\mathrm{SiO}_{2}$ or any combination of $\mathrm{SiO}_{2} / \mathrm{TiO}_{2}$, corresponding to low refractive index and co-sputtered films, respectively. The porosity of $\mathrm{SiO}_{2}$ is limited to $90 \%$, corresponding to a refractive index of 1.05 , which has previously been demonstrated [10]. For each possible design, the largest thicknesses are matched to compositions with the lowest refractive index, and then sorted so that the highindex layers are adjacent to the substrate. This increases the number of designs approximating the optimum antireflection coating - which is expected to have monotonically decreasing refractive index and increasing thickness when moving away from the substrate - and reduces the computation time.

After generating a population of possible coating structures, the fitness of each member of the population is evaluated. The fitness is determined by the reflection coefficient averaged over the wavelength range and angle range of 


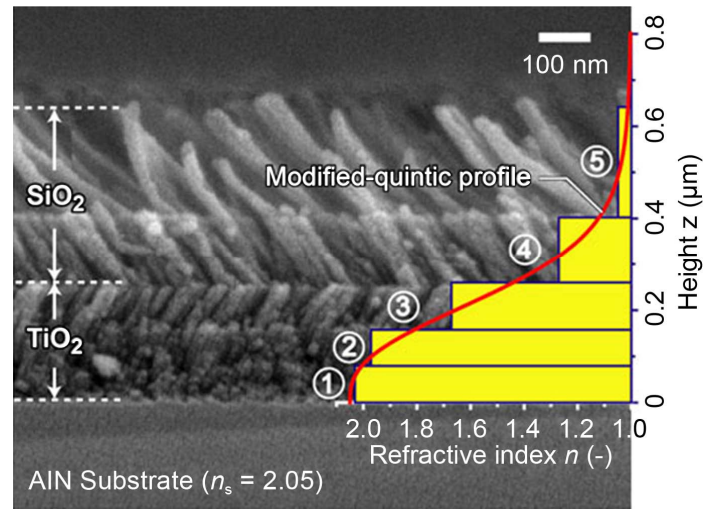

(a)

\begin{tabular}{|l|c|c|c|c|c|}
\hline Layer No. & $\mathbf{1}$ & $\mathbf{2}$ & $\mathbf{3}$ & $\mathbf{4}$ & $\mathbf{5}$ \\
\hline Source & \multicolumn{3}{|c|}{$\mathrm{TiO}_{2}$} & \multicolumn{2}{c|}{$\mathrm{SiO}_{2}$} \\
\hline $\begin{array}{l}\text { Deposition } \\
\text { angle }\end{array}$ & $25^{\circ}$ & $40^{\circ}$ & $65^{\circ}$ & $-68^{\circ}$ & $-87^{\circ}$ \\
\hline $\begin{array}{l}\text { Thickness } \\
\text { (nm) }\end{array}$ & 77.4 & 80.2 & 99.3 & 145.0 & 223.0 \\
\hline $\begin{array}{l}\text { Refractive } \\
\text { index, } \boldsymbol{n}\end{array}$ & 2.03 & 1.97 & 1.67 & 1.27 & 1.05 \\
\hline
\end{tabular}

(b)

Figure 5. (a) Cross-sectional scanning electron micrograph of a $\mathrm{TiO}_{2}$ and $\mathrm{SiO}_{2}$ step-graded index nanowire/nanorod coating which approximates a modified-quintic profile. The graded-index coating consists of three $\mathrm{TiO}_{2}$ nanorod layers and two $\mathrm{SiO}_{2}$ nanorods layers; (b) The physical targets for each layer in the graded index coating, including deposition angle, thickness and refractive index.

interest, $R_{\text {ave }}$, which is given by:

$$
R_{\mathrm{ave}}=\frac{1}{\lambda_{2}-\lambda_{1}} \frac{2}{\pi} \int_{\lambda_{1}}^{\lambda_{2}} \int_{0}^{\pi / 2} \frac{R_{\mathrm{TE}}(\lambda, \theta)+R_{\mathrm{TM}}(\lambda, \theta)}{2} \mathrm{~d} \theta \mathrm{d} \lambda
$$

where $R_{\mathrm{TE}}$ and $R_{\mathrm{TM}}$ are the transverse electric (TE) and transverse magnetic (TM) reflection coefficients. In practice, the fitness function may easily be modified to give greater weight to certain angles of incidence or to certain wavelengths to take into account the responsivity of a particular element. For EO/IR sensor applications, we are particularly interested in optimizing antireflection coating designs for the wavelength range of $250 \mathrm{~nm}$ to $2500 \mathrm{~nm}$ and an incidence angle between $0^{\circ}$ and $40^{\circ}$.

Figure 7 summarizes the simulated performance of an optimized six-layer antireflection structure on a glass substrate. The optimized antireflection structure varies in refractive index between 1.45 and 1.05 , refractive index values which can be obtained with nanostructured $\mathrm{SiO}_{2}$ films deposited by oblique angle deposition. The refractive index profile of the optimized multi-layered antireflection struc-

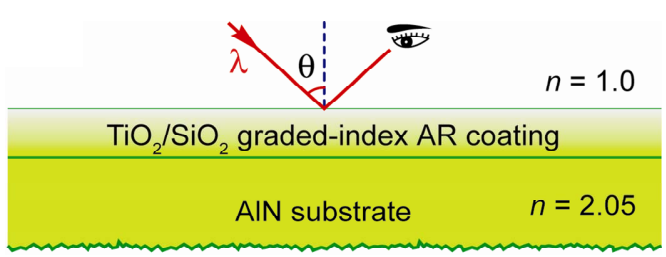

(a)

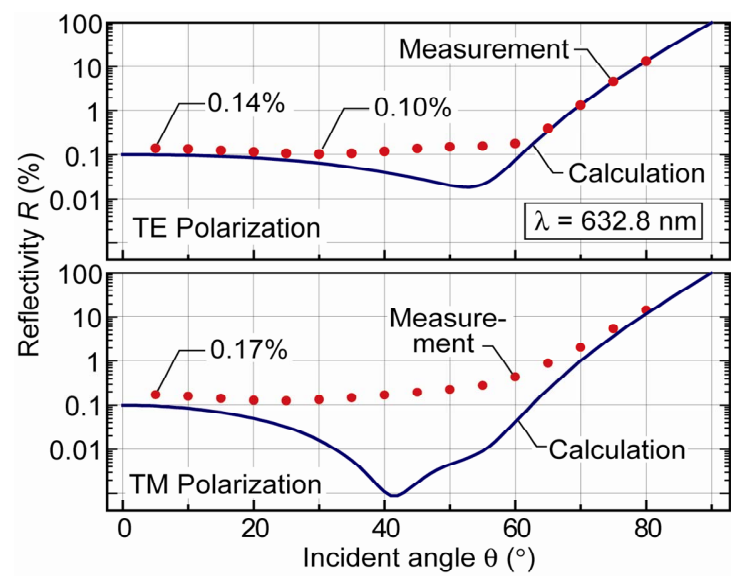

(b)

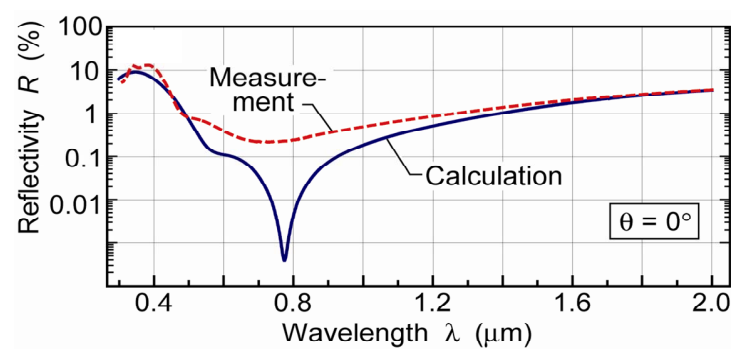

(c)

Figure 6. Reflectivity of a graded-index coating on an AIN substrate, including (a) Schematic of the reflectivity measurement; (b) Theoretical (solid line) and measured (dotted line) reflectivity of polarized light versus incident angle; and (c) Wavelength dependence of theoretical (solid line) and measured (dashed line) reflectivity at normal incidence. Further details can be found in reference [10].

ture approximates a continuously graded refractive index structure with a quintic profile. The potential performance of such a multi-layered, step-graded antireflection structure is quite remarkable. As highlighted in Figure 7(a), normal incidence reflectivity below $0.1 \%$ is projected for a broad spectral band between 0.4 and 1.8 microns. In addition, visible wavelength reflectivity below $0.1 \%$ for both polarization types can be maintained at incident angles between $0^{\circ}$ and $55^{\circ}$, as seen in Figure 7(b).

The potential performance of simpler step-graded antireflection structures with fewer layers has also been modeled and found to be outstanding. Figure 8, for example, compares the calculated reflectance of an uncoated glass substrate to a glass substrate coated with a two-layer structure as a function of wavelength and angle of incidence. 


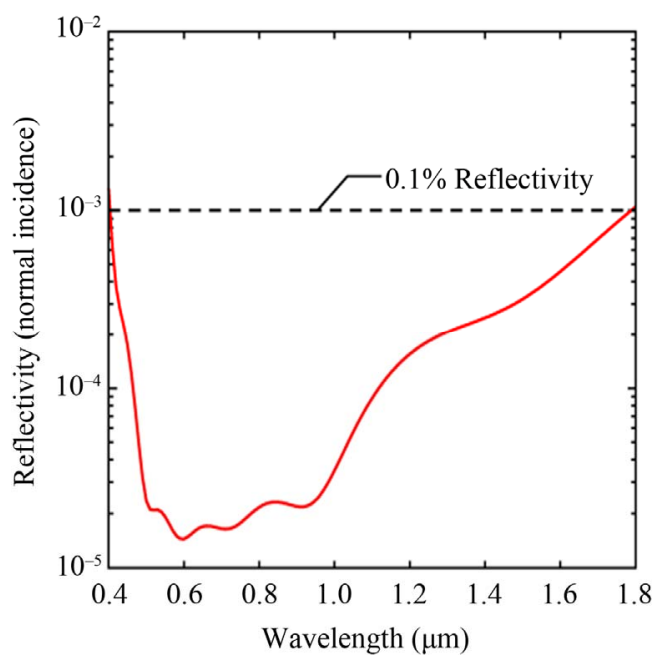

(a)

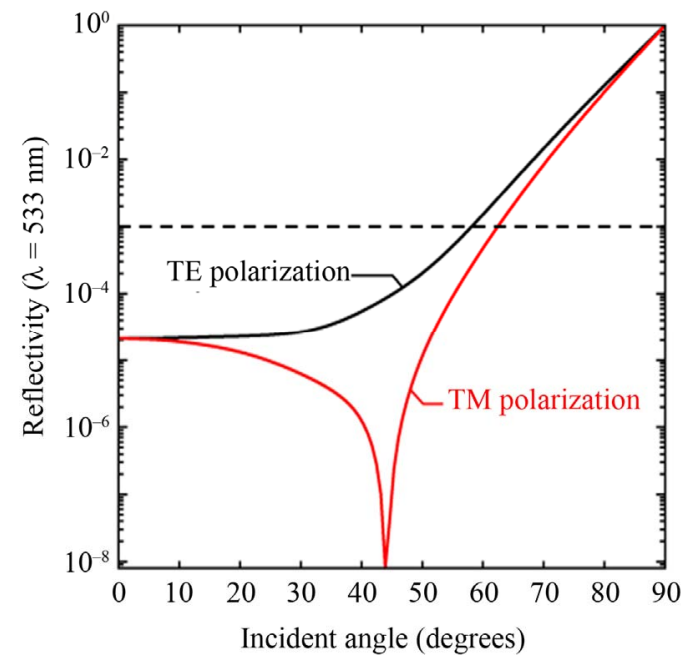

(b)

Figure 7. (a) Simulation of reflectivity from a six-layer, step-graded antireflection structure on a glass window as a function of wavelength between 0.4 and 1.8 microns; and (b) Simulation of reflectivity as a function of polarization for incident angles between $0^{\circ}$ to $90^{\circ}$ for the same six-layer, step-graded antireflection structure.

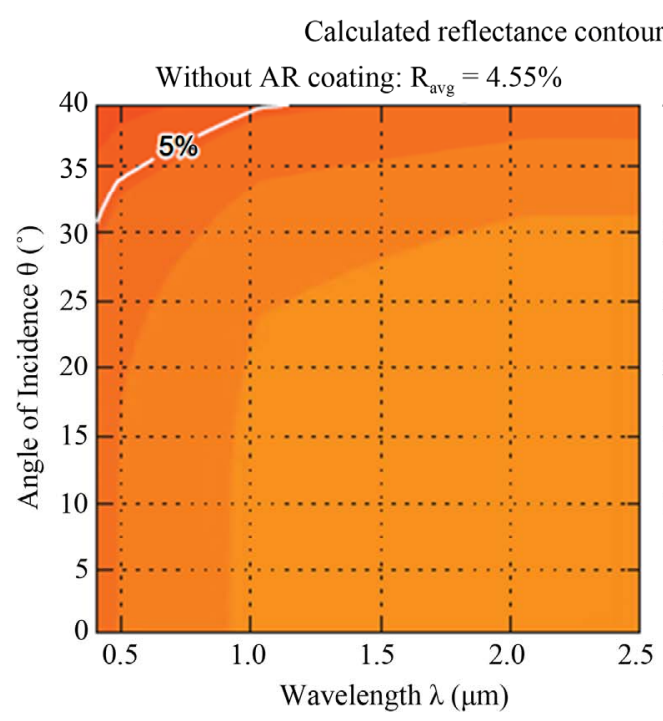

(a)

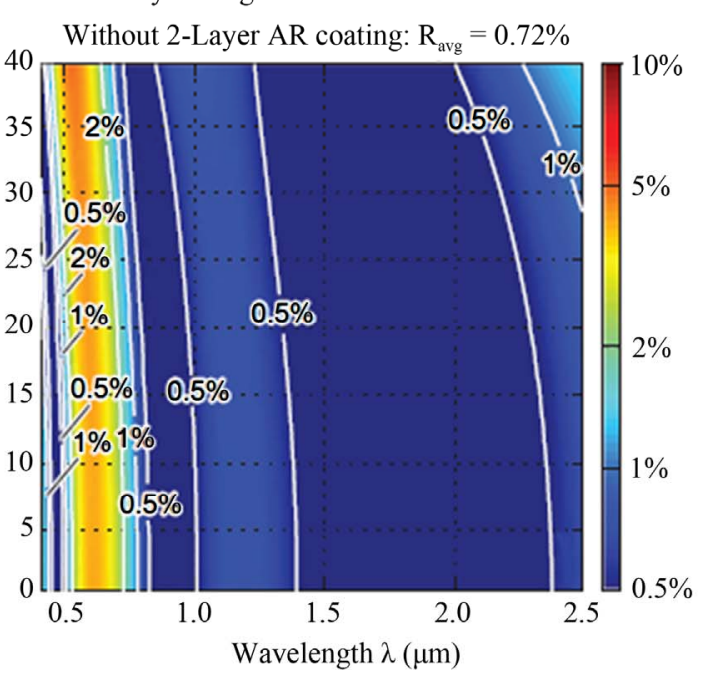

(b)

Figure 8. Contour plots of calculated reflectance versus wavelength and angle of incidence for an infinitely thick glass substrate with (a) No AR coating; and (b) Two-layer step-graded antireflection coating.

The projected reflectance of the two-layer structure is below $1 \%$ for a broad band of wavelengths ranging from the visible to the NIR, and for a wide range on incident angles between $0^{\circ}$ and $40^{\circ}$.

\section{Fabrication and Characterization of Nanostructured Antireflection Coatings on Glass}

We have fabricated and tested a number of different stepgraded antireflection structures on glass substrates [12-14]. In particular, oblique angle deposition has been used to deposit both two- and three-layer structures comprised of nanostructured $\mathrm{SiO}_{2}$. These multi-layer antireflection structures have been deposited both on one and on two sides of a glass substrate, and the transmittance characterized as a function of wavelength and incident angle.

Figure 9 compares the measured broadband performance of an uncoated glass slide to a glass slide coated on two sides with a multi-layered, nanostructured $\mathrm{SiO}_{2}$ coating. The nanostructured coatings were prepared in an electronbeam evaporator using different deposition angles to form distinct layers with a step-graded refractive index profile. The inset in Figure 9 shows a representative crosssectional scanning electron micrograph of a two-layer structure. The transmittance of the coated and uncoated glass 
slides was measured using an angle dependent transmittance measurement setup consisting of a Xenon lamp light source and an Ando AQ6315A optical spectrum analyzer calibrated to detect transmitted photons over a broadband spectrum (400 - $1800 \mathrm{~nm}$ ). The measured peak broadband transmittance at normal incidence of the uncoated glass slide is $92 \%$, in-line with the expected approximate $4 \%$ reflection loss at each glass/air interface. The peak transmittance increases to $98.3 \%$ for the double-sided, nanostructured coated glass, implying an average broadband reflection loss of less than $1 \%$ at each glass/air interface. As shown in Figure 9, the transmittance through the nanostructured $\mathrm{SiO}_{2}$ coated glass is also significantly higher than the uncoated glass across a wide range of incident angles. While the transmittance of the uncoated glass slide falls below $80 \%$ at an incident angle of $65^{\circ}$, the glass slide with the double-sided coating still maintains a transmittance above $95 \%$.

The transmittance of coated and uncoated glass slides has also been measured as a function of wavelength at normal incidence using a JASCO V-570 spectrophotometer. As seen in Figure 10, the measured transmittance through a glass slide is dramatically improved over the entire $400 \mathrm{~nm}$ to $1800 \mathrm{~nm}$ spectrum by the application of a nanostructured antireflection coating. In particular, the average measured broadband transmittance between $350 \mathrm{~nm}$ and $1800 \mathrm{~nm}$ increases from $92.2 \%$ for the uncoated glass, to $98.6 \%$ for the double-sided, nanostructured coated glass. Moreover, the transmittance through the glass coated with a nanostructured $\mathrm{SiO}_{2}$ coating exceeds $97.8 \%$ at all wavelengths between $440 \mathrm{~nm}$ and $1800 \mathrm{~nm}$, implying a glass-air interface reflectivity below $1.1 \%$ over a wide range of wavelengths. Optimized nanostructured antireflection coatings have been shown to outperform an ideal quarter-wavelength $\mathrm{MgF}_{2}$ coating over all wavelengths and incident angles [14].

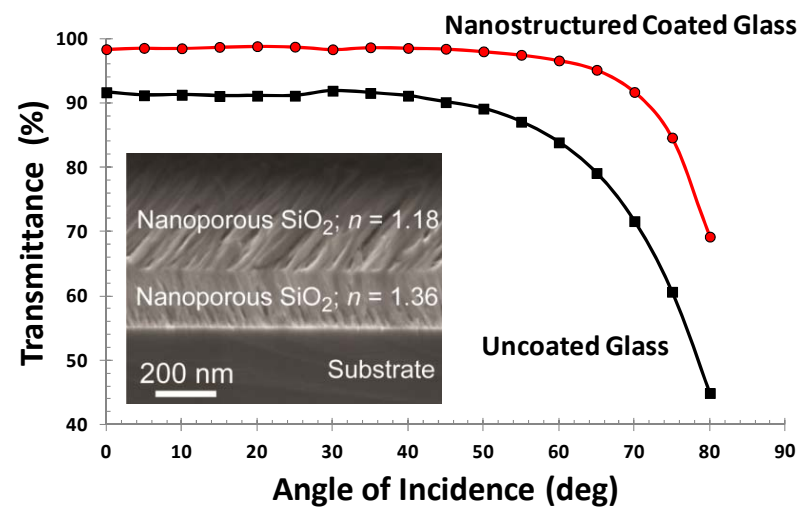

Figure 9. Angle-of-incidence dependent broadband transmittance measurement through a glass slide coated on both sides with a step-graded, nanostructured $\mathrm{SiO}_{2}$ antireflection structure. Also shown is the measured broadband transmittance of an uncoated glass slide, and a cross-sectional scanning electron micrograph of a two-layer nanostructured coating.

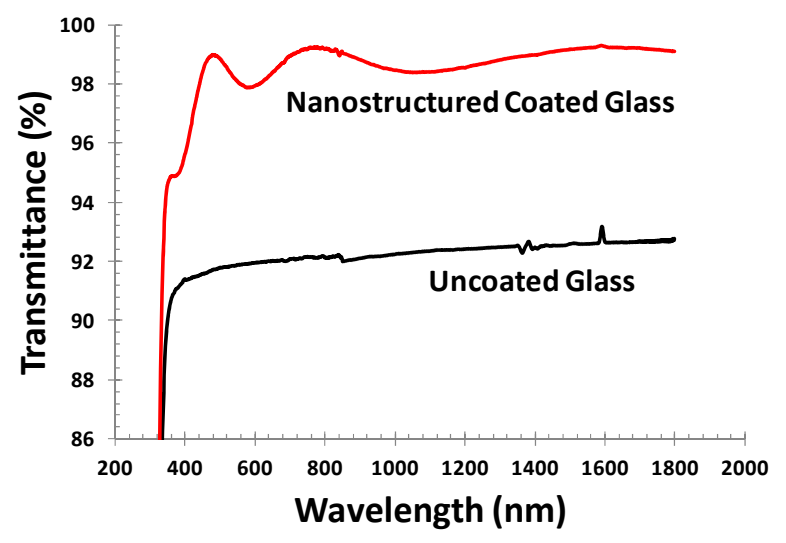

Figure 10. Wavelength dependent transmittance measurement of a step-graded, nanostructured $\mathrm{SiO}_{2}$ antireflection coating on a glass substrate using a JASCO V-570 spectrophotometer.

\section{Summary}

This paper has shown that the growth of oblique angle $\mathrm{SiO}_{2}$ nanowires and nanorods offers an innovative approach for developing high quality antireflection coatings for use on next generation sensors and optical windows to minimize reflection losses for both defense and commercial applications. The antireflection technology discussed in the paper has been shown to be both broadband and omnidirectional in nature. Moreover, we have evaluated antireflection structures that will meet the requirements for a number of applications. Continued efforts are underway to demonstrate nanowire-based antireflection coatings for spectral bands from the UV to the IR for next generation sensors, and to extend their utility to longer wavelength applications.

\section{REFERENCES}

[1] A. K. Sood, R. A. Richwine, Y. R. Puri, S. Horn and R. S. Balcerak, "Design Considerations Using APD Detectors for High Resolution UV Imaging Applications," Proceedings of SPIE, Vol. 7419, 2009, Article ID: 74190V. doi:10.1117/12.829900

[2] M. J. Cohen, T. J. Martin, J. C. Dries and M. J. Lange, "InGaAs Sensor/Focal Plane Arrays for SWIR Applications," Proceedings of SPIE, Vol. 5406, 2004, pp. 38-45.

[3] A. K. Sood, R. A. Richwine, Y. R. Puri, N. DiLello, J. L. Hoyt, T. I. Akinwande, S. Horn, R. S. Balcerak, G. Bulman, R. Venkatasubramanian, A. I. D'Souza, and T. G. Bramhall, "Development of Low Dark Current SiGeDetector Arrays for Visible-NIR Imaging Sensor," Proceedings of SPIE, Vol. 7298, 2009, Article ID: 72983D. doi: $10.1117 / 12.820896$

[4] M. Davis, J. Devitt, M. Greiner, R. Rawe, A. Timlin and D. Wade, "Advanced InSb FPA Sensor Development at CMC Electronics," Proceedings of SPIE, Vol. 5563, 2004, pp. 62-73. doi: $10.1117 / 12.565665$

[5] A. K. Sood, R. A. Richwine, Y. R. Puri, N. K. Dhar, D. L. Polla and P. S. Wijewarnasuriya, "Multispectral EO/IR 
Sensor Model for Evaluating UV, Visible, SWIR, MWIR and LWIR System Performance," Proceedings of SPIE, Vol. 7300, 2009, Article ID: 73000H. doi: $10.1117 / 12.820899$

[6] P. W. Norton, M. Kohin, M. Dovidio and B. Becker, "Commercialization of Uncooled Infrared Technology," Proceedings of SPIE, Vol. 5563, 2004, pp. 55-61. doi:10.1117/12.565663

[7] A. K. Sood, R. A. Richwine, Y. R. Puri, D. L. Polla, N. K. Dhar, Z. L. Wang, J. M. Xu, P. S. Wijewarnasuriya, N. Goldsman, M. B. Soprano and B. Lineberry, "EO/IR Sensors Development Using Zinc Oxide and Carbon Nanostructures," Proceedings of SPIE, Vol. 7318, 2009, Article ID: 731804. doi:10.1117/12.820502

[8] A. K. Sood, Y. R. Puri, L. Becker, M. Z. Tidrow, R. S. Balcerak, G. Brill, P. Wijewarnasuriya, N. Dhar, P. Boieriu, C. Fulk, S. Sivananthan, J. Yehoda and S. Finke, "Development of High-Performance Radiation-Hardened Antireflection Coatings for LWIR and Multicolor IR Focal Plane Arrays," Proceedings of SPIE, Vol. 6206, 2006, Article ID: 620615. doi:10.1117/12.667812

[9] D. J. Poxson, M.-L. Kuo, F. W. Mont, Y.-S. Kim, X. Yan, R. E. Welser, A. K. Sood, J. Cho, S.-Y. Lin and E. F. Schubert, "High-Performance Antireflection Coatings Utilizing Nanoporous Layers," MRS Bulletin, Vol. 36, No. 6, 2011, pp. 434-438. doi:10.1557/mrs.2011.110

[10] J. Q. Xi, M. F. Schubert, J. K. Kim, E. F. Schubert, M. Chen, S.-Y. Lin, W. Liu and J. A. Smart, "Optical Thin-
Film Materials with Low Refractive Index for Broadband Elimination of Fresnel Reflection," Nature Photonics, Vol. 1, 2007, pp. 176-179.

[11] M. F. Schubert, F. W. Mont, S. Chhajed, D. J. Poxson, J. K. Kim and E. Fred Schubert, "Design of Multilayer Antireflection Coatings Made from Co-Sputtered and LowRefractive-Index Materials by Genetic Algorithm," Optics Express, Vol. 16, No. 8, 2008, pp. 5290-5298. doi:10.1364/OE.16.005290

[12] S. Chhajed, D. J. Poxson, X. Yan, J. H. Cho, E. F. Schubert, R. E. Welser, A. K. Sood and J. K. Kim, "Nanostructured Multi-Layer Tailored-Refractive Index AntiReflection Coating for Glass with Broadband and OmniDirectional Characteristics," Applied Physics Express, Vol. 4, 2011, Article ID: 052503.

[13] R. E. Welser, A. W. Sood, A. K. Sood, D. J. Poxson, S. Chhajed, J. Cho, E. F. Schubert, D. L. Polla and N. K. Dhar, "Ultra-High Transmittance through Nanostructured-Coated Glass for Solar Cell Applications," Proceedings of SPIE, Vol. 8035, 2011, Article ID: 80350X. doi: $10.1117 / 12.888129$

[14] R. E. Welser, A. W. Sood, G. G. Pethuraja, A. K. Sood, X. Yan, D. J. Poxson, J. Cho, E. Fred Schubert and J. L. Harvey, "Broadband Nanostructured Antireflection Coating on Glass for Photovoltaic Applications," 38th IEEE Photovoltaic Specialist Conference, Austin, 3-8 June 2012. 